\title{
Open-label Phase II trial to evaluate safety and efficacy of second-line metronomic oral vinorelbine-atezolizumab combination for stage-IV non-small-cell lung cancer - VinMetAtezo trial, (GFPC $\ddagger$ 04-2017)
}

\author{
Alain Vergnenegre ${ }^{1}$, Isabelle Monnet ${ }^{2}$, Acya Bizieux ${ }^{3}$, Marie Bernardi ${ }^{4}$, Anne Marie \\ Chiapa $^{5}$, Hervé Léna ${ }^{5}$, Christos Chouaïd*,2 (iD) \& Gilles Robinet ${ }^{7}$ \\ ${ }^{1}$ UOTC, Centre Hospitalier Universitaire Dupuytren, Limoges, France \\ ${ }^{2}$ Department of Pneumology, Centre Hospitalier Intercommunal Créteil, Créteil, France \\ ${ }^{3}$ Department of Pneumology, Centre Hospitalier Départemental Vendée, La Roche-sur-Yon, France \\ ${ }^{4}$ Department of Pneumology, Centre Hospitalier Aix-en-Provence, Aix-en-Provence, France \\ ${ }^{5}$ Department of Pneumology, Centre Hospitalier Quimper, Quimper, France \\ ${ }^{6}$ Department of Pneumology, Centre Hospitalier Universitaire Rennes, Rennes, France \\ ${ }^{7}$ Department of Pneumology, Centre Hospitalier Universitaire Brest, Brest France \\ *Author for correspondence: Christos.chouaid@chicreteil.fr \\ $\ddagger$ GFPC: French Lung Cancer Group
}

Metronomic chemotherapy is defined as frequent low-dose administration without prolonged drug-free breaks. Combining immune-checkpoint inhibitors and metronomic chemotherapy is a new approach to improve responses and delay onset of resistance to immune-checkpoint inhibitors. This multicenter, Phase II, open-label, single-arm study was designed to assess the safety and efficacy of metronomic oral vinorelbine in combination with immune-checkpoint inhibitors in advanced non-small-cell lung cancers progressing after first-line platinum-based chemotherapy. The recommended metronomic oral vinorelbine dose will be determined during a safety run-in period including 12 patients; the main study will include 59 additional patients. The primary outcome is progression-free survival at 4 months. Secondary outcomes are safety of the combination, median overall survival, objective response rate, disease-control rate at 4 months and quality of life (NCT03801304).

First draft submitted: 18 November 2019; Accepted for publication: 10 December 2019; Published online: 2 January 2020

Keywords: immunotherapy • metronomic chemotherapy • non-small-cell lung cancer • second line

Lung cancer is the leading cause of cancer deaths in USA, with 5 -year survival at $\sim 16 \%$. Representing $>85 \%$ of lung cancers, non-small-cell lung cancer (NSCLC) is the most common type. Unfortunately, most of these patients are diagnosed with locally advanced or metastatic disease [1-4].

Immunotherapy enhanced a paradigmatic shift in NSCLC treatment, especially through the anti-PD1 or its ligand's (PD-1/PD-L1) pathway. PD-1 [3] is an immune-checkpoint receptor expressed by activated T cells. Upon binding to its ligands, PD-L1 and PD-L2, PD-1 normally moderates ongoing immune responses and prevents autoimmunity [3]. Several compounds have been used as second-or-more-line agents [4]. For first-line, chemotherapytreated patients, immunotherapy is a second-line option. In this setting, nivolumab and pembrolizumab (anti-PD-1) and atezolizumab (anti-PD-L1) monoclonal antibodies were approved and have gradually been introduced into clinical management of advanced NSCLCs [5,6].

Despite these notable advances, persistently low objective response rates (ORRs) to second-line immunecheckpoint inhibitors NSCLC treatment [7] have led to new second-line options, especially immunotherapychemotherapy combinations [2]. Second-line immune-checkpoint inhibitors therapy might be combined with chemotherapy to improve immunotherapy efficacy for regimens without major toxicity. Currently, many drugs

Future Medicine 
are combined with immunotherapy as first- or second-line regimens. Oral vinorelbine has been used for many years to treat NSCLCs [8,9]. Metronomic chemotherapy is defined as frequent low-dose administration without prolonged drug-free breaks to reduce toxic effects and prevent rapid revascularization that can promote tumor growth during therapy breaks [9]. The immunostimulatory effects of metronomic chemotherapy are: induced immunogenic cancer-cell death [10]; enhanced antigen presentation through dendritic cell modulation [11] and increased cancer-cell immunogenicity [12]; preferential regulatory T-cell depletion [13]; modulation of myeloidderived suppressor cells [14]; and heightened immune-effector-cell cytotoxic activity, for example, of tumor-specific T cells [15]. Metronomic chemotherapy-immunotherapy synergism has been reported [16].

Using oral vinca alkaloid vinorelbine for metronomic therapy has been accorded considerable attention, especially because of its potent anti-angiogenic and pro-immune and microtubule-targeting properties at low dose [17,18]. Activity was reported in elderly patients against metastatic breast cancer, advanced refractory NSCLCs $[19,20]$ and advanced NSCLCs [21]. Barlesi et al. [22] recently published the usefulness of a mathematical model to determine the optimal metronomic vinorelbine dose for NSCLC and mesothelioma patients. In most studies, the recommended metronomic oral vinorelbine (MOV) dose alone is $50 \mathrm{mg} /$ day, thrice weekly $[19,20,23]$. The MOVE trial, prescribing first-line MOV alone for elderly patients, achieved $18.6 \%$ ORR [23]. Sutiman et al recently comparing two MOV doses combined with erlotinib for NSCLC patients obtained good disease control ( $\sim 50 \%)$ [24]. Safety of MOV combined with sorafenib for advanced NSCLC patients was described previously [25]. More recently the safety of MOV was also confirmed by a retrospective multicentric analysis including 270 advanced NSCLC [26] and an individual patient-data meta-analysis including 9 studies and 418 patients [27].

We describe here the design of a multicenter, Phase II, open-label, single-arm study designed to assess the safety and efficacy of MOV in combination with atezolizumab and the result of a running safety phase. The study hypothesis is based on immunotherapy potentialization by metronomic chemotherapy, herein the atezolizumabMOV combination, whose safety and efficacy have not been assessed previously.

\section{Materials \& Methods}

This multicenter, Phase II, open-label, single-arm study was designed to assess atezolizumab-MOV safety and efficacy in patients with advanced NSCLCs.

Eligibility criteria for inclusion were: advanced NSCLC or relapsed locally advanced NSCLC, without activating $E G F R$ mutation or $A L K$ rearrangement, progressing after first-line platinum-doublet-based chemotherapy, according to RECIST v1.1; a measurable lesion (RECIST v1.1); age $\geq 18$ years, with ECOG PS $<3$ and life expectancy $>12$ weeks; adequate laboratory-test-documented organ-function results during the 3 weeks preceding study enrollment; effective contraception for women of child-bearing potential; national healthcare insurance and written informed consent to participate.

The main noneligibility criteria were: small cell lung, bronchioalveolar or neuroendocrine cancer; known hypersensitivity to immunotherapy; radiotherapy (except for bone or brain) within the 3 months preceding baseline imaging; persistent clinical adverse events (AEs) attributed to prior treatment; active or untreated computed tomography or magnetic resonance imaging detected CNS metastases during screening and prior radiographic assessments; uncontrolled pleural effusion; pericardial effusion; ascites requiring recurrent drainage procedures; uncontrolled/symptomatic hypercalcemia requiring continued bisphosphonate or denosumab use; prior autoimmune disease; human immunodeficiency virus or active hepatitis B- or C-positivity; systemic corticosteroid up to $10 \mathrm{mg} /$ day or other systemic immunosuppressant use during the 2 weeks preceding study enrollment; or anticipated need for systemic immunosuppressant(s) during the trial.

\section{Study design}

A run-in phase aimed to ensure the safety of the fixed-dose atezolizumab (1200 mg iv. on day 1, every 21 days)MOV (40-mg dose, thrice weekly for 3 weeks) combination; the latter was chosen based on the literature [23,25,26]. To assess tolerance, the numbers (\%) of AEs, according to National Cancer Institute CTCAE, will be recorded. Toxicity will be determined by the number (\%) of grade- $\geq 3 \mathrm{AEs}$ in the first 12 patients during the first cycle, with $>20 \%$ grade- $\geq 3$ specific immune-related or oral vinorelbine-related AEs defining dose toxicity. A $\leq 6$-week trial interruption will allow Data Safety Monitoring Board (DMSB) case review.

In the case of toxicity, the dose will be decreased to $30 \mathrm{mg}$, thrice weekly. The same AE-assessment procedure will be applied to the following 12 patients. 


\begin{tabular}{|c|c|c|c|c|}
\hline \multirow[b]{2}{*}{ Adverse event } & \multicolumn{2}{|c|}{ Grades $\leq 2$} & \multicolumn{2}{|c|}{ Grades $>2$} \\
\hline & 1 & 2 & 3 & 4 \\
\hline Hematologic & 1 & 1 & - & - \\
\hline Infectious & 1 & & & \\
\hline Neurologic & - & 1 & & \\
\hline Vascular thrombus & - & 2 & & \\
\hline Fatigue & & 2 & & \\
\hline Digestive & 5 & 3 & & \\
\hline General disorders & - & 3 & $2^{\dagger}$ & - \\
\hline Pain & - & 1 & $1^{\dagger}$ & - \\
\hline Cutaneous & 1 & 1 & & \\
\hline
\end{tabular}

\section{The main study}

Once the safety run-in phase has been completed and the regimen validated, the study design will be as follows: MOV ( 40 or $30 \mathrm{mg}$, thrice weekly for 3 weeks) in combination with fixed atezolizumab infusions (1200 mg iv. on day 1, every 21 days). Patients will be treated until disease progression. When progression according to RECIST criteria occurs, MOV will be stopped. Atezolizumab will be continued until clinical progression. Overall, 71 patients will be enrolled in this Phase II study, including the 12 safety run-in-phase participants.

Cross-sectional analyses will attempt to identify relevant biomarkers and evaluate their relationship(s) to clinical outcomes. The specific analysis will be based on PD-L1 expression, but other biomarkers would be tested.

\section{Outcomes}

The primary outcome is PFS rate at 4 months. The secondary outcomes are median PFS, median OS, tolerance according CTAE, ORR, disease-control rate and quality of life during the study using the EORTC QLQ-C30 scale.

\section{Statistical analyses}

This open-label, multicenter, Phase II study used the exact single-stage Phase II design defined by A'hern [28]. The sample size is based on an exact binomial distribution.

Minimal efficacy hypothesis (p1) is set at 55\% event-free rate of PFS at 4 months; (p0), indicating that the strategy is clearly ineffective, is set at $40 \%$ PFS at 4 months (based on the OAK study's $43 \%$ PFS at 4 months). With a $5 \%$ alpha-risk (unilateral perspective) and a $20 \% \beta$-risk, the number of assessable subjects is set at 71 . The Phase III trial threshold is 36 successes/71 subjects, with success defined as a subject without death or progression at 4 months. The percentage of patient successes at 4 months will be described with its $95 \%$ confidence interval, estimated by the exact method. $\mathrm{p}<0.05$ will define statistical significance. The intent-to-treat population (all patients included) will be analyzed. The per-protocol population will also be analyzed. The results will be presented according to the CONSORT Statement recommendations.

The statistical analysis plan will be validated by the trial's Scientific Committee.

\section{Ethical considerations}

The study will be conducted in compliance with the principles of the Declaration of Helsinki, and each participating institution's Institutional Review Board has approved the protocol. All patients must give written informed consent before any screening or inclusion procedures. The regulatory authority approved the protocol on 24 October 2018 and the Ethics Committee on 22 November 2018 (NCT03801304, EudraCT number: 2018-000164-28).

\section{Results}

The trial was opened in four centers for the run-in phase (25 January 2019 to 19 March 2019). 12 patients were included: the DMSB reviewed 25 AEs (Table 1). Three grade- 3 AEs occurred: deteriorated general condition attributed to increased size of brain metastases, not treatment-related; metastasis-related bone pain, not treatment- 
related, that was rapidly attenuated with specific treatment and without affecting the protocol; loss of appetite and weight loss, possibly treatment-related, leading to protocol interruption for 1 week.

The DMSB recommended transition to the main study at the $40-\mathrm{mg} / \mathrm{day}$, thrice weekly, MOV dose on 16 April 2019, which was validated by the regulatory authority.

\section{Discussion \& conclusion}

The safety and efficacy study of the atezolizumab-MOV combination will enable us to determine whether metronomic chemotherapy administration can enhance the efficacy of immunotherapy alone with an acceptable tolerance profile. Depending on market-access times, the majority of patients diagnosed with advanced NSCLC are or will be eligible for first-line combination immunotherapy-platinum-doublet chemotherapy regimens, but most of them will progress on them. No management recommendations for second-or-more lines exist for these patients but the possibility of immunotherapy-metronomic chemotherapy reintroduction, after a therapeutic pause or after second-line chemotherapy is an alternative option worth exploring. This Phase II trial will enable safety and efficacy evaluations of the atezolizumab-MOV combination.

\section{Executive summary}

Background

- Atezolizumab, a monoclonal antibody targeting PD1-L1, is approved for second-line treatment of non-small-cell lung cancers.

- The concept of metronomic chemotherapy is defined as frequent, low-dose administration without prolonged drug-free breaks.

- New options for second-line therapy of non-small-cell lung cancers are needed.

- Combining immune-checkpoint inhibitors and metronomic chemotherapy is such a new approach to improve responses and retard development of resistance to immune-checkpoint inhibitors.

Trial design

Open-label Phase II trial to evaluate safety and efficacy of second-line metronomic oral vinorelbine-atezolizumab combination for stage-IV non-small-cell lung cancer

\section{Disclaimer}

Sponsors were involved in the study design, but not in patient inclusion, collection and interpretation of data, writing the report and the decision to submit the article for publication. This multicenter Phase II, open-label, single-arm study was designed to assess the safety and efficacy of the metronomic oral vinorelbine and atezolizumab combination.

Financial \& competing interests disclosure

The study was sponsored and funded by Laroche Company and Pierre-Fabre Oncology Company. A Vergnenegre reported travel, grant and honorariums from AZ, Roche, MSD, BMS, Pierre Fabre; Isabelle MONNET travel from Roche; H Lena reported travel, grant and honorariums from AZ, Roche, MSD, BMS; Christos CHOUAID grant, travel, honorariums from Roche Pierre Fabre Oncologie AstraZeneca, BMS, MSD Bayer; Gilles Robinet reported grant, travel, honorariums from Roche Pierre Fabre Oncologie AstraZeneca, BMS, MSD Bayer Acya Bizieux. The authors have no other relevant affiliations or financial involvement with any organization or entity with a financial interest in or financial conflict with the subject matter or materials discussed in the manuscript apart from those disclosed.

No writing assistance was utilized in the production of this manuscript.

\section{Ethical conduct of research}

The authors state that they have obtained appropriate institutional review board approval or have followed the principles outlined in the Declaration of Helsinki for all human or animal experimental investigations. In addition, for investigations involving human subjects, informed consent has been obtained from the participants involved.

\section{Open access}

This work is licensed under the Attribution-NonCommercial-NoDerivatives 4.0 Unported License. To view a copy of this license, visit http://creativecommons.org/licenses/by-nc-nd/4.0/ 


\section{References}

Papers of special note have been highlighted as: $\bullet$ of interest; $\bullet \bullet$ of considerable interest

1. Novello S, Barlesi F, Califano R et al. Metastatic non-small-cell lung cancer: ESMO clinical practice guidelines for diagnosis, treatment and follow-up. Ann. Oncol. 27(Suppl. 5), v1-v27 (2016).

2. Karachaliou N, Sosa AE, Barron FB, Gonzalez Cao M, Santarpia M, Rosell R. Pharmacological management of relapsed/refractory NSCLC with chemical drugs. Expert Opin. Pharmacother. 18(3), 295-304 (2017).

3. Harvey RD. Immunologic and clinical effects of targeting PD-1 in lung cancer. Clin. Pharmacol. Ther. 96(2), 14-23 (2014).

4. Bustamante Alvarez JG, Gonzalez-Cao M, Karachaliou N et al. Advances in immunotherapy for treatment of lung cancer. Cancer Biol. Med. 12(3), 209-222 (2015).

5. Schvartsman G, Ferrarotto R, Massarelli E. Checkpoint inhibitors in lung cancer: latest developments and clinical potential. Ther. Adv. Med. Oncol. 8(6), 460-473 (2016).

6. Chae YK, Pan A, Davis AA et al. Recent advances and future strategies for immune-checkpoint inhibition in small-cell lung cancer. Clin. Lung Cancer 18(2), 132-140 (2017).

7. Vansteenkiste J, Wauters E, Park K, Rittmeyer A, Sandler A, Spira A. Prospects and progress of atezolizumab in non-small cell lung cancer. Expert Opin.Biol. Ther. 17(6), 781-789 (2017).

8. Marty M, Fumoleau P, Adenis A et al. Oral vinorelbine pharmacokinetics and absolute bioavailability study in patients with solid tumors. Ann. Oncol. 12(11), 1643-1649 (2001).

- Pharmacokinetics and bioavailability of oral vinorelbine.

9. Andre N, Carre M, Pasquier E. Metronomics: towards personalized chemotherapy? Nat. Rev. Clin. Oncol. 11(7), 413-431 (2014).

- Concept of metronomic chemotherapy.

10. Tesniere A, Apetoh L, Ghiringhelli F et al. Immunogenic cancer cell death: a key- lock paradigm. Curr. Opin. Immunol. 20(5), 504-511 (2008).

11. Kaneno R, Shurin GV, Tourkova IL, Shurin MR. Chemomodulation of human dendritic cell function by antineoplastic agents in low noncytotoxic concentrations. J. Transl. Med. 7, 58 (2009).

12. Kaneno R, Shurin GV, Kaneno FM, Naiditch H, Luo J, Shurin MR. Chemotherapeutic agents in low noncytotoxic concentrations increase immunogenicity of human colon cancer cells. Cell Oncol. 34(2), 97-106 (2011).

-• Immunogenicity of metronomic chemotherapy in cancer cells.

13. Ghiringhelli F, Menard C, Puig PE et al. Metronomic cyclophosphamide regimen selectively depletes CD4+CD25+ regulatory T cells and restores T and NK effector functions in end stage cancer patients. Cancer Immunol. Immunother. 56(5), 641-648 (2007).

14. Michels T, Shurin GV, Naiditch H, Sevko A, Umansky V, Shurin MR. Paclitaxel promotes differentiation of myeloid-derived suppressor cells into dendritic cells in vitro in a TLR4-independent manner. J. Immunotoxicol. 9(3), 292-300 (2012).

15. Sierro SR, Donda A, Perret R et al. Combination of lentivector immunization and low-dose chemotherapy or PD-1/PD-L1 blocking primes self-reactive T cells and induces anti-tumor immunity. Eur. J. Immunol. 41(8), 2217-2228 (2011).

16. Barbolosi D, Ciccolini J, Meille C et al. Metronomics chemotherapy: time for computational decision support. Cancer Chemother. Pharmacol. 74(3), 647-652 (2014).

17. Pasquier E, Andre N, Braguer D. Targeting microtubules to inhibit angiogenesis and disrupt tumour vasculature: implications for cancer treatment. Curr Cancer Drug Targets 7(6), 566-581 (2007).

18. Galano G, Caputo M, Tecce MF, Capasso A. Efficacy and tolerability of vinorelbine in the cancer therapy. Curr Drug Saf. 6(3), 185-193 (2011)

19. Kontopodis E, Hatzidaki D, Varthalitis I et al. A Phase II study of metronomic oral vinorelbine administered in the second line and beyond in non-small cell lung cancer (NSCLC): a Phase II study of the Hellenic Oncology Research Group. J. Chemother. 25(1), 49-55 (2013).

20. Rajdev L, Negassa A, Dai Q, Goldberg G, Miller K, Sparano JA. Phase I trial of metronomic oral vinorelbine in patients with advanced cancer. Cancer Chemother. Pharmacol. 68(5), 1119-1124 (2011).

21. Guetz S, Tufman A, von Pawel J et al. Metronomic treatment of advanced non-small-cell lung cancer with daily oral vinorelbine - a phase I trial. Onco Targets Ther. 10, 1081-1089 (2017).

22. Barlesi F, Imbs DC, Tomasini P et al. Mathematical modelling for phase I cancer trials: a study of metronomic vinorelbine for advanced non-small cell lung cancer (NSCLC) and mesothelioma patients. Oncotarget 8(29), 47161-47166 (2017).

23. Camerini A, Puccetti C, Donati S et al. Metronomic oral vinorelbine as first-line treatment in elderly patients with advanced non-small cell lung cancer: results of a phase II trial (MOVE trial). BMC Cancer 15, 359 (2015).

-. Efficacy of metronomic oral vinorelbine in advanced non-small-cell lung cancer.

24. Sutiman N, Zhang Z, Tan EH et al. Phase I study of oral vinorelbine in combination with erlotinib in advanced non-small cell lung cancer (NSCLC) using two different schedules. PLoS ONE 11(5), e0154316 (2016). 
25. Tan EH, Tan DS, Li WY et al. Metronomic vinorelbine (oral) in combination with sorafenib in advanced non-small cell lung cancer. Lung Cancer 88(3), 289-296 (2015).

26. Camerini A, Banna GL, Cinieri S. Metronomic oral vinorelbine for the treatment of advanced non-small cell lung cancer: a multicenter international retrospective analysis. Clin. Transl. Oncol. 21(6), 790-795 (2019).

-. Efficacy of metronomic oral vinorelbine in advanced non-small-cell lung cancer.

27. Pujol JL, Coffy A, Camerini A et al. An individual patient-data meta-analysis of metronomic oral vinorelbine in metastatic non-small cell lung cancer. PLoS ONE 14(8), e0220988 (2019).

28. A'Hern RP. Sample size tables for exact single-stage phase II designs. Stat. Med. 20(6), 859-866 (2001). 